\title{
Short communication: Glutamine modulates inflammatory responses to lipopolysaccharide in ex vivo bovine endometrium
}

\author{
Pablo G. Noleto, ${ }^{*} \dagger^{1}$ João Paulo E. Saut, ${ }^{*}$ and I. Martin Sheldon† \\ *Large Animal Health Laboratory, Federal University of Uberlândia, Uberlândia, 38400-902, Brazil \\ †Institute of Life Science, Swansea University Medical School, Swansea, SA2 8PP, United Kingdom
}

\section{ABSTRACT}

Bacteria infect the endometrium lining the uterus of cattle after parturition, and clearance of these microbes depends on a robust innate immune response to bacterial molecules, such as the endotoxin lipopolysaccharide (LPS). Endometrial inflammation is characterized by secretion of the cytokines IL- $1 \beta$ and IL- 6 and the chemokine IL-8. However, animals often fail to clear invading bacteria and develop uterine disease if they are in negative energy balance, with reduced abundance of glucose and glutamine, which are substrates for energy in tissues. Depletion of glucose blunts inflammatory responses in the endometrium, but the role of glutamine is not clear. The present study tested the hypothesis that depletion of glutamine compromises inflammatory responses to LPS in endometrial tissue. Ex vivo organ cultures of endometrium were challenged with LPS, and culture supernatants accumulated IL$1 \beta$, IL-6, and IL-8, as expected. However, reducing the availability of glutamine in culture medium containing glucose reduced the accumulation of IL-1 $\beta$, IL-6, and IL- 8 by $>50 \%$. Surprisingly, in the absence of glucose, supplying increasing amounts of glutamine was not sufficient to augment inflammatory responses to LPS, whereas, in the absence of glutamine, supplying more glucose increased inflammation. Furthermore, inhibiting glycolysis reduced the accumulation of IL-1 $\beta$, IL-6, and IL- 8 by $>50 \%$, even when glutamine and glucose were abundant. In conclusion, depletion of glutamine reduces inflammatory responses to LPS in the endometrium, and the activity of glutamine depends on glucose and glycolysis. These data provide mechanistic insights into how negative energy balance may be linked to postpartum uterine disease.

Key words: cow, uterus, innate immunity, metabolism, inflammation

\footnotetext{
Received September 20, 2016.

Accepted December 3, 2016.

${ }^{1}$ Corresponding author: noleto@ufu.br
}

\section{Short Communication}

Postpartum uterine disease in dairy cows costs $\$ 600$ million/yr in the United States because of treatment, reduced milk production, and replacement of infertile animals (Sheldon et al., 2009). Uterine disease is associated with metabolic stress in dairy cows, which compromises inflammatory responses to bacterial infections of the uterus after parturition (Esposito et al., 2014). Understanding how metabolism and immunity are integrated is important. We have already established that glucose deprivation reduces the inflammatory response to the bacterial endotoxin LPS in ex vivo organ cultures of bovine endometrium (Turner et al., 2016). However, little is known about how glutamine, the other major energy substrate, affects innate immunity in the endometrium.

The uterus of dairy cattle is always contaminated with bacteria after parturition (Sheldon et al., 2002; Santos et al., 2011). Clearance of these bacteria from the endometrium lining the uterus depends on a rapid and robust innate immune response, including the secretion of cytokines, such as IL-1 $\beta$ and IL-6, and chemokines, such as IL-8, which attract neutrophils to the site of infection (Hammon et al., 2006; Cronin et al., 2012). Endometrial innate immunity depends on host cell pattern recognition receptors binding pathogen-associated molecular patterns, such as LPS from Escherichia coli (Borges et al., 2012; Cronin et al., 2012; Saut et al., 2014; Turner et al., 2014). However, negative energy balance, with reduced availability of glucose and glutamine, is associated with perturbation of the immune response in cattle (Hammon et al., 2006; Wathes et al., 2009) and persistence of bacterial infections in the endometrium, leading to uterine disease (Giuliodori et al., 2013; Ingvartsen and Moyes, 2013; Esposito et al., 2014).

The innate immune response is energetically expensive for immune cells and endometrial tissue (Turner et al., 2016). The 2 main fuels for cellular energy are glucose and glutamine (Newsholme, 2001; Vander Heiden et al., 2011). Glucose generates ATP via glycolysis and 
the Krebs cycle, and anaplerosis of glutamine replenishes Krebs cycle intermediates (DeBerardinis and Cheng, 2010). However, cows often have reduced abundance of glucose and glutamine in the peripheral circulation after parturition (Doepel et al., 2006; Ingvartsen and Moyes, 2013). We previously established that depletion of glucose in the presence of glutamine reduces the secretion of IL-1 $\beta$, IL-6, and IL-8 from organ cultures of bovine endometrium (Turner et al., 2016). Notably, the abundance of glucose, and not the concentration of glucose, was vital to cellular responses. However, the effect of glutamine abundance on endometrial inflammation is unknown. The present study tested the hypothesis that depletion of glutamine compromises inflammatory responses to LPS in endometrial tissue

To explore the role of glutamine in innate immunity, we prepared ex vivo organ cultures of bovine endometrium, as described previously (Borges et al., 2012; Saut et al., 2014). Briefly, uteri with no evidence of genital disease or microbial infection were collected from cattle after slaughter and processed as part of the normal work of an abattoir. Organ cultures were collected from the endometrium using sterile 8-mmdiameter biopsy punches (Stiefel Laboratories Ltd., High Wycombe, UK), immediately placed in 24-well plates (TPP, Trasadingen, Switzerland) containing 1 $\mathrm{mL}$ of culture medium/well, and incubated at $37^{\circ} \mathrm{C}$ in a humidified atmosphere of air with $5 \% \mathrm{CO}_{2}$. To determine whether glutamine was required for inflammatory responses to LPS, endometrium was cultured for $24 \mathrm{~h}$ in custom-made serum-free culture media containing a range of amounts of glucose (0-0.9 mg/organ), freshly prepared glutamine $(0-0.584 \mathrm{mg} /$ organ $)$, or both and challenged with vehicle or $100 \mathrm{ng} / \mathrm{mL}$ ultrapure LPS from E. coli O111:B4 (InvivoGen, Toulouse, France). The highest amounts of glutamine and glucose were those typically used in bovine endometrial organ and cell culture (Borges et al., 2012; Turner et al., 2014). The media were prepared by adding glucose (Sigma, Gillingham, UK; G8644), glutamine (Sigma; G7513), or both to glucose-free, glutamine-free Dulbecco's modified Eagle's medium (Thermo Fisher Scientific, Paisley, UK; A1443001). The role of glycolysis was investigated by adding $50 \mathrm{mM}$ 2-deoxy-D-glucose (Sigma; D8375) to the media; 2-deoxy-D-glucose is an inhibitor of glycolysis because replacing the D-glucose 2-hydroxyl group with hydrogen prevents glycolysis (Tannahill et al., 2013).

At the end of each experiment, supernatants were collected and stored at $-20^{\circ} \mathrm{C}$ for subsequent analyses, and organ weights were recorded. Concentrations of IL$1 \beta$ and IL-6 in supernatants were measured in duplicate by ELISA according to the manufacturer's instructions (Bovine IL-1 $\beta$ Screening Set ESS0027, Thermo Fisher
Scientific; Bovine IL-6 DuoSet DY8190, R\&D Systems, Abingdon, UK); bovine IL-8 was measured by ELISA as described previously (Cronin et al., 2015). The interand intraassay coefficients of variation were all $<10 \%$, and the limits of detection were $13 \mathrm{pg} / \mathrm{mL}$ for IL- $1 \beta, 75$ $\mathrm{pg} / \mathrm{mL}$ for IL-6, and $32 \mathrm{pg} / \mathrm{mL}$ for IL-8. Lactate dehydrogenase (LDH) activity was measured using an $\mathrm{LDH}$ activity assay (Cambridge Bioscience Ltd., Cambridge, UK) to evaluate tissue damage, as previously described (Borges et al., 2012; Turner et al., 2016). Data were normalized for the weight of each organ culture and presented as mean (SEM). Each experiment was performed using endometrial organ cultures from at least 3 independent animals, with 2 technical replicates for each treatment. Statistical analyses were performed using GraphPad Prism (GraphPad Software, La Jolla, CA), with the animal as the experimental unit. Comparisons among treatments were evaluated using ANOVA with Sidak's multiple comparisons test, and significance was ascribed when $P<0.05$.

To determine whether glutamine could modulate tissue inflammatory responses, ex vivo organ cultures of endometrium were cultured in a range of amounts of glutamine up to $0.584 \mathrm{mg} /$ organ, in the presence of $0.36 \mathrm{mg}$ of glucose/organ, and challenged with vehicle or LPS for $24 \mathrm{~h}$. As expected, the culture supernatants accumulated more IL-1 $\beta$, IL-6, and IL-8 in response to LPS than to vehicle (ANOVA, $P<0.001$; Figure $1 \mathrm{~A}-\mathrm{C})$. However, limiting glutamine availability reduced the accumulation of IL-1 $\beta$, IL- 6 , and IL- 8 in response to LPS, and the absence of glutamine reduced inflammatory responses by $>50 \%$ (ANOVA, $P<0.01$; Figure $1 \mathrm{~A}-\mathrm{C})$. To explore whether the presence of glucose was required for glutamine to affect inflammatory responses to LPS, endometrium was cultured in media containing the same range of amounts of glutamine, but in the absence of glucose, and challenged with vehicle or LPS. The culture supernatants accumulated more IL-1 $\beta$, IL-6, and IL-8 in response to LPS than vehicle (ANOVA, $P<0.001$; Figure $1 \mathrm{D}-\mathrm{F}$ ), but the maximal concentrations were lower than when glucose was present (Figure 1A-C). Surprisingly, in the absence of glucose, supplying increasing amounts of glutamine did not significantly augment the accumulation of IL$1 \beta$ (ANOVA, $P=0.93)$, IL-6 $(P=0.73)$, or IL-8 $(P=$ 0.94 ) in response to LPS (Figure 1D-F). One concern might be that deficits in the availability of glucose and glutamine might influence tissue viability, so we used the accumulation of LDH in supernatants as a marker of tissue viability, as described previously (Borges et al., 2012; Turner et al., 2016). Even the absence of glucose and glutamine for $24 \mathrm{~h}$ did not significantly affect LDH accumulation $(12.5 \pm 2.7$ vs. $19.0 \pm 1.3 \mathrm{mU}$ of $\mathrm{LDH} /$ $\mathrm{mg}$ of tissue in 0 vs. $0.584 \mathrm{mg}$ of glutamine, $\mathrm{n}=3$ ). 


\section{$0.36 \mathrm{mg} / \mathrm{organ} \mathrm{Glucose}$}

A

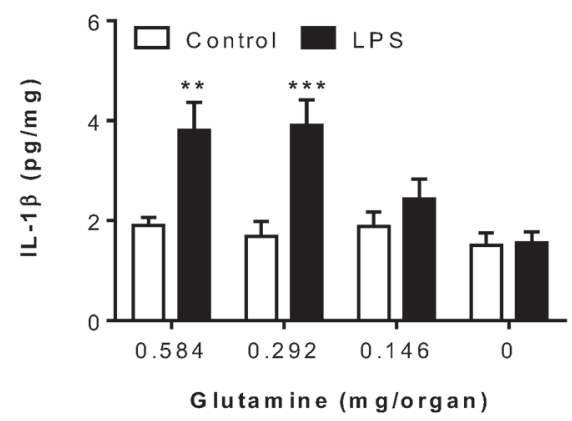

No Glucose

D

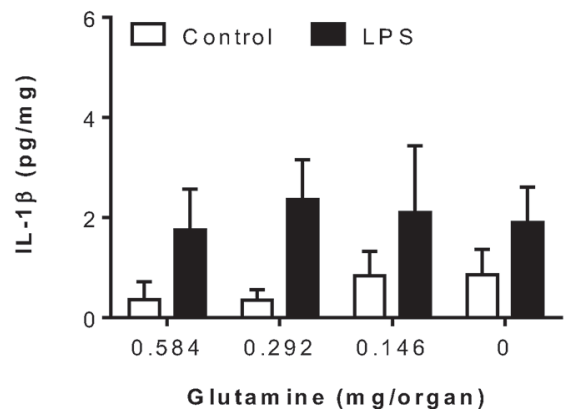

B

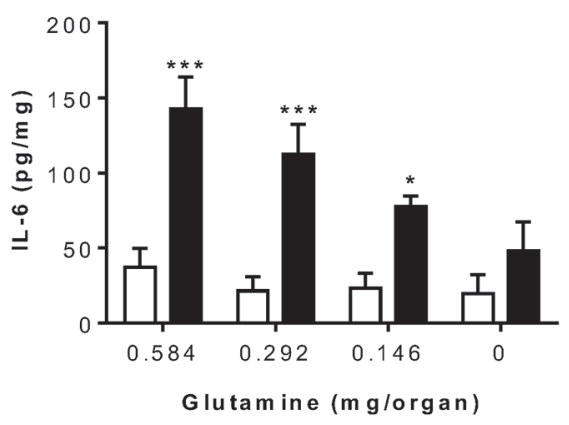

E

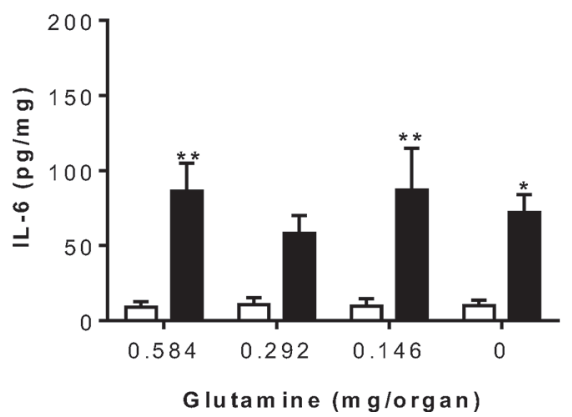

C

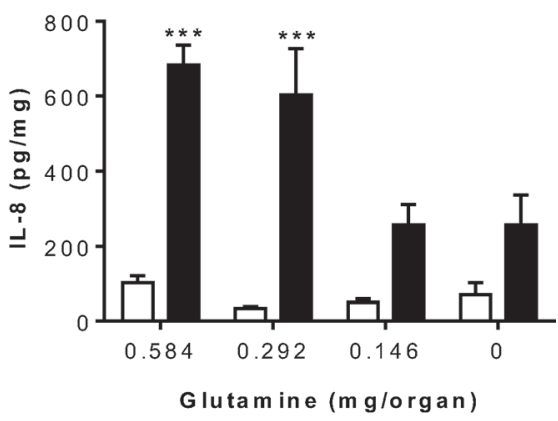

$\mathrm{F}$

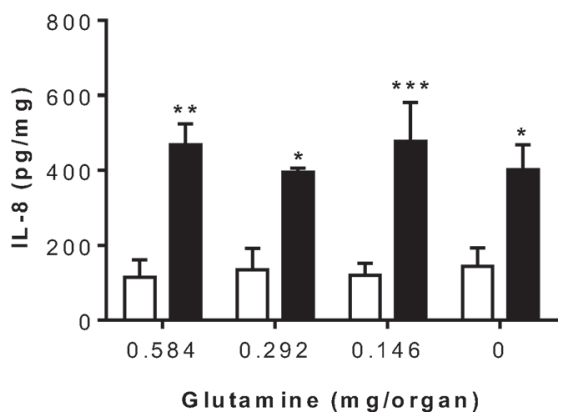

Figure 1. Glutamine modulates inflammatory responses to LPS. Ex vivo organ cultures of bovine endometrium were cultured for $24 \mathrm{~h}$ in medium containing the indicated amounts of glutamine, with $0.36 \mathrm{mg}$ of glucose $(\mathrm{A}-\mathrm{C})$ or no glucose (D-F), and challenged with control vehicle $(\square)$ or $100 \mathrm{ng} / \mathrm{mL}$ LPS $(\square)$. At the end of the experiment, organ weights were recorded (35.8 $\pm 11.2 \mathrm{mg})$ and the accumulation of IL-1 $\beta$ (A, D), IL-6 (B, E), and IL-8 (C, F) was measured in supernatants. Data are presented as mean \pm SEM concentration per milligram of tissue from 4 independent animals and analyzed by ANOVA with Sidak's multiple comparisons test; values differ from control: $* * * P<0.001,{ }^{* *} P<0.01$, $* P<0.05$.

Together, these data provide evidence that glutamine availability influences tissue inflammatory responses, but glucose may be required for glutamine to modulate inflammatory responses to LPS.

We previously showed that limiting glucose availability reduced the accumulation of IL-1 $\beta$, IL- 6 , and IL- 8 in response to LPS by $>45 \%$ when ex vivo organ cultures of bovine endometrium were cultured with $0.584 \mathrm{mg}$ of glutamine/organ (Turner et al., 2016). To explore whether the presence of glutamine was essential, endometrium was cultured in media containing a range of amounts of glucose in the absence of glutamine and challenged with vehicle or LPS for $24 \mathrm{~h}$. The culture supernatants accumulated more IL-1 $\beta$, IL-6, and IL-8 in response to LPS than vehicle (ANOVA, $P<0.001$; Figure 2). However, the absence of glutamine did not affect the expected increased accumulation of IL-1 $\beta$, IL-6, and IL-8 in response to LPS when more glucose was supplied in the medium (ANOVA, $P<0.05$; Figure
2). Taken together, these data support the idea that glucose availability influences tissue inflammatory responses, and they provide evidence that this effect does not require the presence of glutamine.

To understand why glutamine depends on the presence of glucose for inflammatory responses to LPS, we considered the role of glucose in glycolysis. To explore the role of glycolysis, 2-deoxy-D-glucose was used to inhibit glycolysis when endometrium was treated with glutamine, with or without glucose (Tannahill et al., 2013). As expected, culture supernatants supplied with glucose or with glucose and glutamine accumulated more IL-1 $\beta$, IL-6, and IL- 8 in response to LPS than vehicle (ANOVA, $P<0.01$; Figure 3 ). Inflammatory responses to LPS were reduced by at least $50 \%$ when glycolysis was inhibited in endometrium supplied with glucose alone, as might be anticipated (ANOVA, $P<$ 0.01; Figure 3). However, the inhibition of inflammation by 2-deoxy-D-glucose was not rescued by the addition 
A

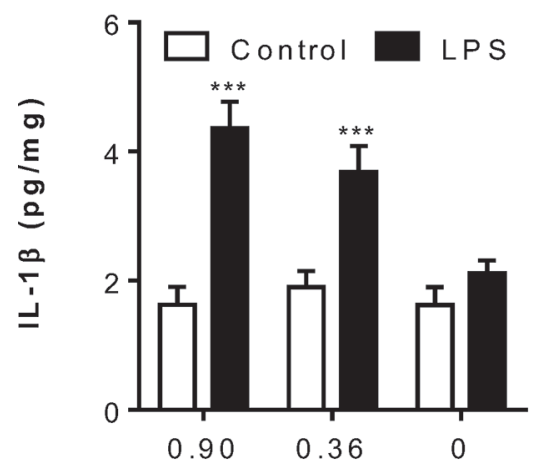

Glucose (mglorgan)
B

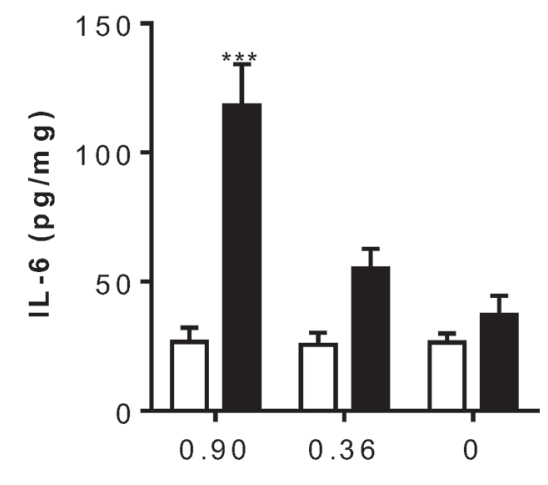

Glucose (mg/organ)
C

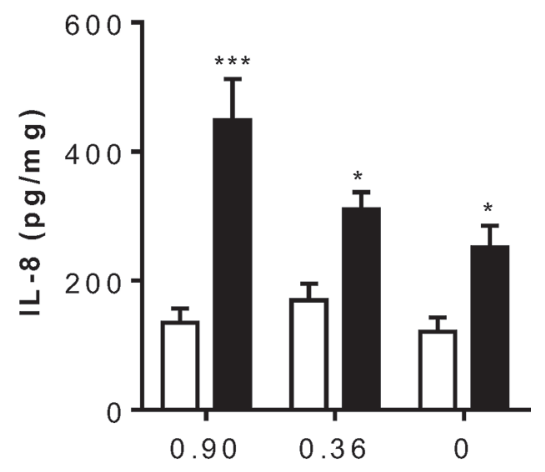

Glucose (mg/organ)

Figure 2. Glucose modulates inflammatory responses to LPS even in the absence of glutamine. Ex vivo organ cultures of endometrium were cultured for $24 \mathrm{~h}$ in medium containing $0.9,0.36$, or $0 \mathrm{mg}$ of glucose, without glutamine, and challenged with control vehicle ( $\square$ ) or $100 \mathrm{ng} / \mathrm{mL}$

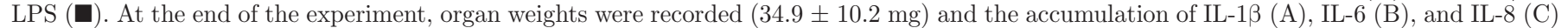
measured in supernatants. Data are presented as mean \pm SEM concentration per milligram of tissue from 7 independent animals and analyzed by ANOVA with Sidak's multiple comparisons test; values differ from control: ${ }^{* * *} P<0.001,{ }^{*} P<0.05$.

of glutamine, as well as glucose. These data provide evidence that the effect of glutamine on inflammation is dependent on glycolysis.

Deficits in glucose and glutamine are typical of postpartum cattle in negative energy balance, and this metabolic stress is associated with uterine disease (Wathes et al., 2009; Giuliodori et al., 2013; Esposito et al., 2014). Deficits in glucose abundance perturb innate immunity in the endometrium (Turner et al., 2016). In the present study, we provide evidence that limiting glutamine availability also perturbs the inflammatory response to LPS in endometrial tissue. However, to our surprise, the presence of glucose was necessary for glutamine supplementation to augment endometrial in-
A

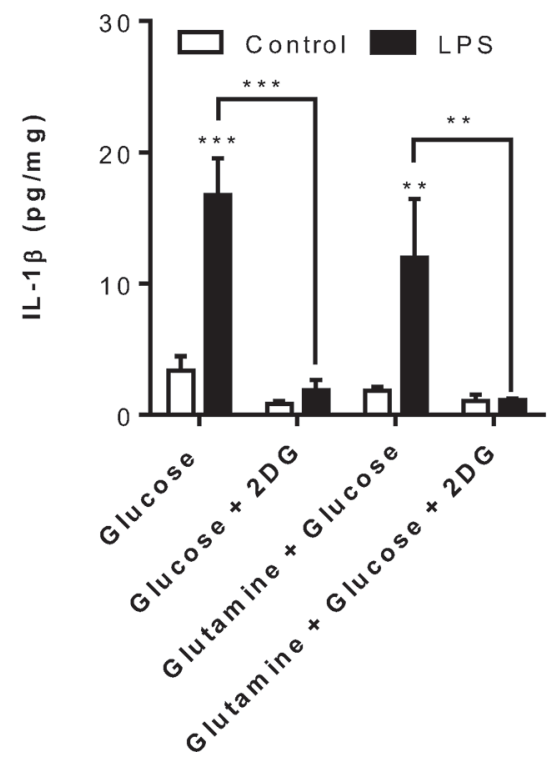

B

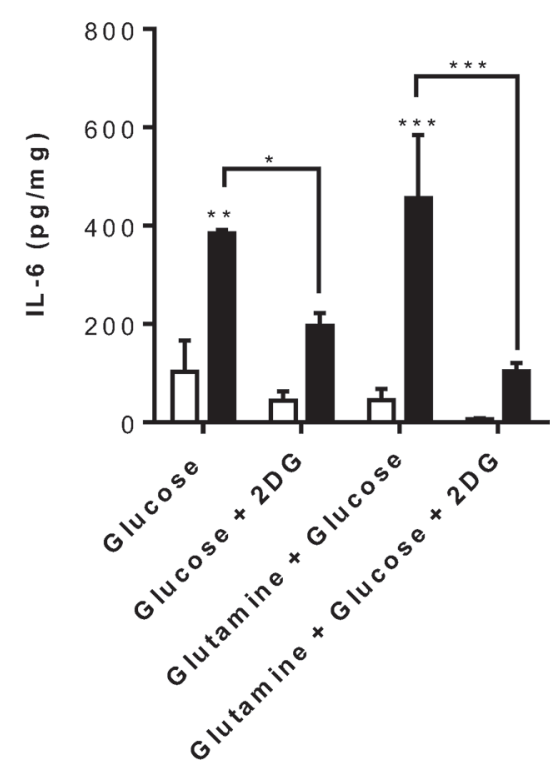

c

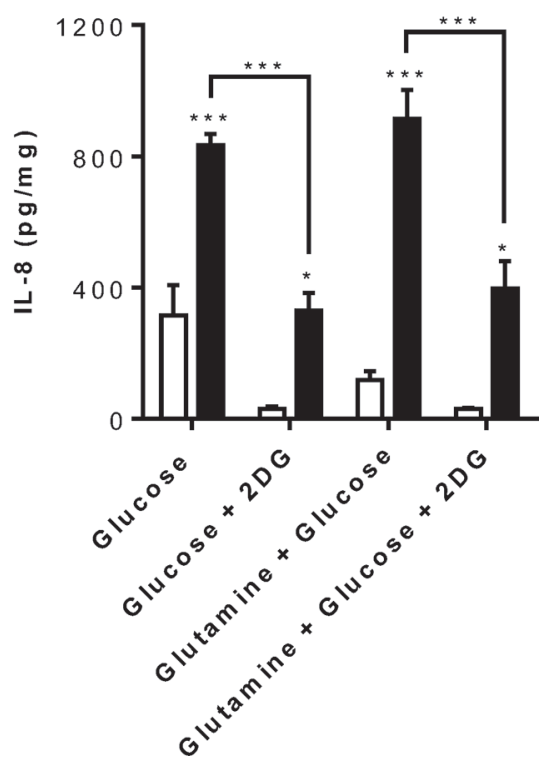

Figure 3. Glycolysis is important for inflammatory responses to LPS. Ex vivo organ cultures of endometrium were cultured for $24 \mathrm{~h}$ in medium containing $0.584 \mathrm{mg}$ of glucose/organ with or without $50 \mathrm{~m} M$ 2-deoxy-D-glucose (2DG) to inhibit glycolysis, or $0.584 \mathrm{mg}$ of glutamine/ organ and $0.36 \mathrm{mg}$ of glucose/organ with or without $50 \mathrm{mM} 2 \mathrm{DG}$, and challenged with control vehicle ( $\square$ ) or $100 \mathrm{ng} / \mathrm{mL} \mathrm{LPS} \mathrm{( \square ).} \mathrm{At} \mathrm{the} \mathrm{end}$ of the experiment, organ weights were recorded $(29.8 \pm 8.9 \mathrm{mg})$ and the accumulation of IL-1 $\beta$ (A), IL-6 (B), and IL-8 (C) measured in supernatants. Data are presented as mean \pm SEM concentration per milligram of tissue from 3 independent animals and analyzed by ANOVA with Sidak's multiple comparisons test; values differ from control: ${ }^{* * *} P<0.001,{ }^{* *} P<0.01,{ }^{*} P<0.05$ or where indicated. 
flammation. Furthermore, inhibiting glycolysis reduced the accumulation of IL-1 $\beta$, IL- 6 , and IL- 8 , even when glutamine and glucose were abundant.

The optimal inflammatory responses for postpartum endometrial health are not clear; for example, development of uterine disease has been associated with both increased and reduced expression of $I L 1 B$ mRNA (Herath et al., 2009; Galvão et al., 2011). Inflammation aims to restore tissue homeostasis in the face of infection but requires a complex balance between resistance and damage (Medzhitov, 2008); inadequate immune responses may fail to counter infections, while overexuberant or persistent inflammation cause immunopathology. However, negative energy balance is consistently associated with perturbation of the immune response in cattle (Hammon et al., 2006; Wathes et al., 2009). Exploring the links between metabolism and immunity in the present study, we found that limiting glutamine availability perturbed the inflammatory response to LPS in endometrial tissue. Glucose and glutamine are the main substrates that cells and tissues use to generate energy via glycolysis, the Krebs cycle, and anaplerosis (Newsholme, 2001; Vander Heiden et al., 2011). Inflammatory responses are energetically expensive, presumably because tissues need to generate inflammatory mediators and repair damaged cells. So, limiting glutamine is likely to reduce carbon and nitrogen sources for synthesis of inflammatory mediators as well as limit the abundance of Krebs cycle intermediates for production of ATP. Glutamine, via glutamate, usually yields $\alpha$-ketoglutarate in mitochondria, but in some cells the $\gamma$-aminobutyric acid (GABA) shunt also produces succinate for the Krebs cycle (Tannahill et al., 2013). However, it was surprising that the presence of glucose and glycolysis were necessary for glutamine to augment endometrial inflammation. We suggest that metabolites of glycolysis are necessary to fully exploit the anapleurosis derived from glutamine in the endometrium. Indeed, glycolysis has additional importance during bacterial infections because cellular responses to LPS induce the Warburg effect, in which metabolism is diverted away from the Krebs cycle and toward glycolysis (Tannahill et al., 2013; Turner et al., 2016). Tracing of metabolites may be necessary to determine the precise pathways by which cells link glutamine to inflammation.

The present study provides evidence of how glutamine may affect innate immunity in tissues. However, it was notable that, although much reduced, depletion of both glucose and glutamine did not completely abrogate the inflammatory response to LPS. It is likely that tissues catabolize other nutrients as energy, carbon, and nitrogen sources to synthesize cytokines and chemokines. Translation of the observations in the present study will require further studies of how glutamine affects endometrial biology and immunity. Given that the endometrium comprises multiple types of cell, it would be interesting to determine whether the effect of glutamine is a generalized mechanism or associated with specific cells.

In conclusion, depletion of glutamine reduced inflammatory responses to LPS in ex vivo organ cultures of bovine endometrium in the present study. However, metabolic support for inflammation provided by supplying glutamine depended on the presence of glucose and glycolysis. These data provide a mechanistic insight into how negative energy balance may be linked to postpartum uterine disease.

\section{ACKNOWLEDGMENTS}

The work was funded by a project grant to IMS by the Biotechnology and Biological Sciences Research Council (BB/I017240/1; Swindon, UK). Coordenacao de Aperfeicoamento de Pessoal de Nivel Superior (99999.010219/2014-05; Brasilia, Brazil) and Conselho Nacional de Desenvolvimento Cientifico e Tecnologico (486143/2013-9; Brasilia, Brazil) were supported PGN and JPES. We thank James Cronin, Matthew Turner, and Luisa Carneiro (Swansea University) for training and advice.

\section{REFERENCES}

Borges, A. M., G. D. Healey, and I. M. Sheldon. 2012. Explants of intact endometrium to model bovine innate immunity and inflammation ex vivo. Am. J. Reprod. Immunol. 67:526-539.

Cronin, J. G., R. Hodges, S. Pedersen, and I. M. Sheldon. 2015. Enzyme linked immunosorbent assay for quantification of bovine interleukin-8 to study infection and immunity in the female genital tract. Am. J. Reprod. Immunol. 73:372-382.

Cronin, J. G., M. L. Turner, L. Goetze, C. E. Bryant, and I. M. Sheldon. 2012. Toll-like receptor 4 and MYD88-dependent signaling mechanisms of the innate immune system are essential for the response to lipopolysaccharide by epithelial and stromal cells of the bovine endometrium. Biol. Reprod. 86:51-59.

DeBerardinis, R. J., and T. Cheng. 2010. Q's next: The diverse functions of glutamine in metabolism, cell biology and cancer. Oncogene 29:313-324.

Doepel, L., M. Lessard, N. Gagnon, G. E. Lobley, J. F. Bernier, P. Dubreuil, and H. Lapierre. 2006. Effect of postruminal glutamine supplementation on immune response and milk production in dairy cows. J. Dairy Sci. 89:3107-3121.

Esposito, G., P. C. Irons, E. C. Webb, and A. Chapwanya. 2014. Interactions between negative energy balance, metabolic diseases, uterine health and immune response in transition dairy cows. Anim. Reprod. Sci. 144:60-71.

Galvão, K. N., N. R. Santos, J. S. Galvão, and R. O. Gilbert. 2011. Association between endometritis and endometrial cytokine expression in postpartum Holstein cows. Theriogenology 76:290-299.

Giuliodori, M. J., R. P. Magnasco, D. Becu-Villalobos, I. M. LacauMengido, C. A. Risco, and R. L. de la Sota. 2013. Metritis in dairy cows: Risk factors and reproductive performance. J. Dairy Sci. 96:3621-3631.

Hammon, D. S., I. M. Evjen, T. R. Dhiman, J. P. Goff, and J. L. Walters. 2006. Neutrophil function and energy status in Holstein 
cows with uterine health disorders. Vet. Immunol. Immunopathol. 113:21-29.

Herath, S., S. T. Lilly, N. R. Santos, R. O. Gilbert, L. Goetze, C. E. Bryant, J. O. White, J. Cronin, and I. M. Sheldon. 2009. Expression of genes associated with immunity in the endometrium of cattle with disparate postpartum uterine disease and fertility. Reprod. Biol. Endocrinol. 7:55.

Ingvartsen, K. L., and K. Moyes. 2013. Nutrition, immune function and health of dairy cattle. Animal 7(Suppl. 1):112-122.

Medzhitov, R. 2008. Origin and physiological roles of inflammation. Nature 454:428-435.

Newsholme, P. 2001. Why is L-glutamine metabolism important to cells of the immune system in health, postinjury, surgery or infection? J. Nutr. 131(Suppl.):2515S-2522S.

Santos, T. M., R. O. Gilbert, and R. C. Bicalho. 2011. Metagenomic analysis of the uterine bacterial microbiota in healthy and metritic postpartum dairy cows. J. Dairy Sci. 94:291-302.

Saut, J. P., G. D. Healey, A. M. Borges, and I. M. Sheldon. 2014. Ovarian steroids do not affect bovine endometrial cytokine or chemokine responses to Escherichia coli or LPS in vitro. Reproduction 148:593-606.

Sheldon, I. M., J. Cronin, L. Goetze, G. Donofrio, and H. J. Schuberth. 2009. Defining postpartum uterine disease and the mechanisms of infection and immunity in the female reproductive tract in cattle. Biol. Reprod. 81:1025-1032.

Sheldon, I. M., D. E. Noakes, A. N. Rycroft, D. U. Pfeiffer, and H. Dobson. 2002. Influence of uterine bacterial contamination after parturition on ovarian dominant follicle selection and follicle growth and function in cattle. Reproduction 123:837-845.
Tannahill, G. M., A. M. Curtis, J. Adamik, E. M. Palsson-McDermott A. F. McGettrick, G. Goel, C. Frezza, N. J. Bernard, B. Kelly, N. H. Foley, L. Zheng, A. Gardet, Z. Tong, S. S. Jany, S. C. Corr, M. Haneklaus, B. E. Caffrey, K. Pierce, S. Walmsley, F. C. Beasley, E. Cummins, V. Nizet, M. Whyte, C. T. Taylor, H. Lin, S. L. Masters, E. Gottlieb, V. P. Kelly, C. Clish, P. E. Auron, R. J. Xavier, and L. A. O'Neill. 2013. Succinate is an inflammatory signal that induces IL-1beta through HIF-1alpha. Nature 496:238-242.

Turner, M. L., J. C. Cronin, G. D. Healey, and I. M. Sheldon. 2014 Epithelial and stromal cells of bovine endometrium have roles in innate immunity and initiate inflammatory responses to bacterial lipopeptides in vitro via Toll-like receptors TLR2, TLR1 and TLR6. Endocrinology 155:1453-1465.

Turner, M. L., J. G. Cronin, P. G. Noleto, and I. M. Sheldon. 2016. Glucose availability and AMP-activated protein kinase link energy metabolism and innate immunity in the bovine endometrium. PLoS One 11:e0151416.

Vander Heiden, M. G., S. Y. Lunt, T. L. Dayton, B. P. Fiske, W. J. Israelsen, K. R. Mattaini, N. I. Vokes, G. Stephanopoulos, L. C. Cantley, C. M. Metallo, and J. W. Locasale. 2011. Metabolic pathway alterations that support cell proliferation. Cold Spring Harb. Symp. Quant. Biol. 76:325-334.

Wathes, D. C., Z. Cheng, W. Chowdhury, M. A. Fenwick, R. Fitzpatrick, D. G. Morris, J. Patton, and J. J. Murphy. 2009. Negative energy balance alters global gene expression and immune responses in the uterus of postpartum dairy cows. Physiol. Genomics 39:1-13. 